\title{
Triply heavy baryons and heavy quark spin symmetry
}

\author{
J. M. Flynn, ${ }^{1}$ E. Hernández, ${ }^{2}$ and J. Nieves ${ }^{3}$ \\ ${ }^{1}$ School of Physics and Astronomy, University of Southampton, Highfield, Southampton SO17 1BJ, United Kingdom \\ ${ }^{2}$ Departamento de Física Fundamental e IUFFyM, Universidad de Salamanca, E-37008 Salamanca, Spain \\ ${ }^{3}$ Instituto de Física Corpuscular (IFIC), Centro Mixto CSIC-Universidad de Valencia, \\ Institutos de Investigación de Paterna, Apartado 22085, E-46071 Valencia, Spain
}

(Received 13 October 2011; published 11 January 2012)

\begin{abstract}
We study the semileptonic $b \rightarrow c$ decays of the lowest-lying triply heavy baryons made from $b$ and $c$ quarks in the limit $m_{b}, m_{c} \gg \Lambda_{\mathrm{QCD}}$ and close to the zero-recoil point. The separate heavy-quark spin symmetries strongly constrain the matrix elements, leading to single form factors for $c c b \rightarrow c c c$, $b b c \rightarrow c c b$, and $b b b \rightarrow b b c$ baryon decays. We also study the effects on these systems of using a $Y$-shaped confinement potential, as suggested by lattice QCD results for the interaction between three static quarks.
\end{abstract}

DOI: 10.1103/PhysRevD.85.014012

PACS numbers: 12.39.Hg, 12.39.Jh, 13.30.Ce, 14.20.Mr

\section{INTRODUCTION}

Triply heavy baryons are systems of great theoretical interest, since they may serve to help us better understand the interaction among heavy quarks in an environment free of valence light quarks. Besides, being baryonic analogues of heavy quarkonium, they might yield sharp tests for QCD. Studying these baryons will be also very useful for understanding the three quark static potential.

With no experimental information available on these systems, previous studies have concentrated on their spectrum. To our knowledge the first such study was carried out in 1980 [1] using a QCD-motivated bag model (BM). A mass formula was derived by Bjorken in Ref. [2] providing predictions for the masses that were larger than those found in Ref. [1]. In Ref. [2] the possibility for discovery of the $\Omega_{c c c}$ state was also discussed. More recently there have been other phenomenological mass determinations that include nonrelativistic constituent quark model (NRCQM) calculations [3-5], the relativistic three quark model (RTQM) evaluation of Ref. [6], or the Regge approach in Ref. [7]. More fundamental approaches to the subject include the potential nonrelativistic QCD (pNRQCD) studies of Refs. [8,9] or the QCD sum rule (QCDSR) evaluation of Ref. [10]. In Ref. [11] the leading order (LO) pNRQCD result of Ref. [8] is used, ${ }^{1}$ while a mass calculation that includes next-to-next-to-leading order within the same framework has just appeared [12]. The mass of the triply heavy baryon $\Omega_{b b b}$ has been also recently calculated in lattice QCD (LQCD) using $2+1$ flavors of light sea quarks [13].

Triply charmed baryon production in the $e^{+} e^{-}$reaction was analyzed in Ref. [14] with the result that the predicted production rate was very small. Better perspectives for production are expected at the LHC due to its high lumi-

\footnotetext{
${ }^{1}$ This coincides with the $1 / r$, or Coulomb, interaction that comes from one-gluon exchange.
}

nosity. First estimates of the cross section production at the LHC were evaluated in Refs. [15-18]. A recent evaluation [19] finds that around $10^{4}-10^{5}$ events of triply heavy baryons, with $c c c$ and $c c b$ quark content, can be accumulated for $10 \mathrm{fb}$ of integrated luminosity. The authors of this last work conclude that it is quite likely triply heavy baryons would be discovered at the LHC. With this in mind, study of their properties beyond spectroscopy seems timely.

In this work, we will study the lowest-lying $\left(J^{\pi}=1 / 2^{+}\right.$, $3 / 2^{+}$) triply heavy baryons composed of $b$ and $c$ quarks. ${ }^{2}$ Heavy-quark spin symmetry (HQSS) is of particular interest to study these systems. HQSS is an approximate symmetry of QCD in the limit $m_{b}, m_{c} \gg \Lambda_{\mathrm{QCD}}$, and has proved to be an extremely useful tool when dealing with heavy hadrons [20-22]. This symmetry amounts to the decoupling of the heavy-quark spins [20,21]. In Ref. [23] it is argued that this symmetry cannot be considered as asymptotically valid in heavy-heavy states, since the momentum exchange between two heavy quarks might be much larger than $\Lambda_{\mathrm{QCD}}$, in sharp contrast to the situation for heavy-light systems. For mesons with two equal-mass heavy quarks, Ref. [23] argues that the hyperfine splitting $\Delta m$ scales as $\Delta m \approx m_{Q} \alpha_{s}^{4}\left(m_{Q}\right)$ for sufficiently large heavy-quark mass $m_{Q}$ and thus asymptotically increases with $m_{Q}$. However, $\frac{\Delta m}{m} \approx \alpha_{s}^{4}\left(m_{Q}\right)$ still approaches zero as $m_{Q}$ tends to infinity and the hyperfine splitting becomes negligible compared to the total mass. Moreover, the linear behavior is estimated in Ref. [23] to take over from the $\alpha_{s}^{4}\left(m_{Q}\right)$ logarithmic falloff for heavy-quark masses in the region of $10 \mathrm{GeV}$. Hence for systems with two heavy quarks, with masses below $10 \mathrm{GeV}$, HQSS should still be valid. That being the case, HQSS should be a useful

\footnotetext{
${ }^{2}$ In what follows we will denote by $\Xi$ the baryons with spin $1 / 2$, while we will use $\Xi^{*}$ and $\Omega^{*}$ for the spin $3 / 2$ ones.
} 
approximate symmetry to address baryons made out of three $c$ and/or $b$ heavy quarks, as we aim to do in this work.

The study of baryons requires the solution of the threebody problem. In the past we have made extensive use of HQSS constraints and have developed a simple variational scheme to find masses and wave functions of single [24] and double [25] heavy baryons. We have used the resulting wave functions to study their semileptonic $b \rightarrow c$ [25-28] and $c \rightarrow s, d$ [29] decays. The separate heavy-quark spin symmetries strongly constrain the matrix elements and, in the limit $m_{b}, m_{c} \gg \Lambda_{\mathrm{QCD}}$ and close to the zero-recoil point, they lead to single form factors for all these decays.

Here, we extend our scheme to study triply heavy baryons. We derive for the first time HQSS relations for their semileptonic $b \rightarrow c$ decays from which we can make approximate, but model independent, predictions for some decay width ratios. We give absolute values of the semileptonic $b \rightarrow c$ decay widths, as well. We also study the effects in these baryons of considering a LQCD inspired three-body confinement potential (denoted as $Y$ in [1]) instead of the commonly used one, obtained from the sum of two-body quark-quark terms.

\section{SPIN SYMMETRY}

We will consider decays induced by the semileptonic weak decay of a $b$ quark to a $c$ quark. Near the zero-recoil point, the velocities of the initial and final baryons are approximately the same. If the momenta of the initial and final baryons are $p_{\mu}=m v_{\mu}$ and $p_{\mu}^{\prime}=m^{\prime} v_{\mu}^{\prime}=$ $m^{\prime} v_{\mu}+k_{\mu}$, respectively, then $k$ will be a small residual momentum near the zero-recoil point. For the initial baryon at rest we have that $k \cdot v=E^{\prime}-m^{\prime}$. For a small final momentum this is approximately given by $\vec{p}^{\prime 2} / 2 m^{\prime}$ and then is $\mathcal{O}\left(1 / \mathrm{m}^{\prime}\right)$ close to zero recoil. We will work near zero recoil and thus neglect $v \cdot k$ below.

The consequences of spin symmetry for weak matrix elements can be derived using the "trace formalism" $[22,30]$. The scheme advocated here is similar to that employed in Ref. [31] to study the semileptonic $b c$ to $c c$ baryon decays. To represent baryons with three heavy quarks we will use wave functions comprising tensor products of Dirac matrices and spinors. For $Q_{1} Q_{1} Q_{2}$ baryons containing two heavy quarks $Q_{1}$ and a distinct heavy-quark $Q_{2}$, we have

$$
\begin{aligned}
\Xi_{Q_{1} Q_{1} Q_{2}}= & {\left[\frac{(1+\not)}{2} \gamma_{\mu} \frac{(1-\not)}{2}\right]_{\alpha \beta} } \\
& \times\left[\frac{1}{\sqrt{3}}\left(v^{\mu}+\gamma^{\mu}\right) \gamma_{5} u(v, r)\right]_{\gamma}, \\
\Xi_{Q_{1} Q_{1} Q_{2}}^{*}= & {\left[\frac{(1+\not)}{2} \gamma_{\mu} \frac{(1-\not)}{2}\right]_{\alpha \beta} u_{\gamma}^{\mu}(v, r), }
\end{aligned}
$$

where we have indicated Dirac quark indices $\alpha, \beta$, and $\gamma$ explicitly on the right-hand sides and $r$ is a helicity label for the baryon. ${ }^{3}$ For the $\Xi^{*}$ states, $u_{\gamma}^{\mu}(v, r)$ is a RaritaSchwinger spinor. For the baryon containing three heavy quarks of the same flavor, we use

$$
\Omega_{Q Q Q}^{*}=\frac{1}{\sqrt{3}}\left[\frac{(1+\not)}{2} \gamma_{\mu} \frac{(1-\not)}{2}\right]_{\alpha \beta} u_{\gamma}^{\mu}(v, r) .
$$

These wave functions can be considered as matrix elements of the form $\left\langle 0\left|Q_{1 \alpha} \bar{Q}_{1 \beta}^{c} Q_{2 \gamma}\right| B_{Q_{1} Q_{1} Q_{2}}\right\rangle$ where $\bar{Q}^{c}=$ $Q^{T} C$ with $C$ the charge-conjugation matrix. In each case we couple two quarks of the same flavor in a symmetric spin-1 state in the first factor and combine with a spinor for the third quark. Under a Lorentz transformation, $\Lambda$, and heavy-quark spin transformations $S_{Q}$, a wave function of the form $\Gamma_{\alpha \beta} \mathcal{U}_{\gamma}$, with $\mathcal{U}=\frac{1}{\sqrt{3}}\left(v^{\mu}+\gamma^{\mu}\right) \gamma_{5} u$ or $u^{\mu}$, transforms as

$$
\begin{aligned}
& \Gamma \mathcal{U} \rightarrow S(\Lambda) \Gamma S^{-1}(\Lambda) S(\Lambda) \mathcal{U}, \\
& \Gamma \mathcal{U} \rightarrow S_{Q_{1}} \Gamma S_{Q_{1}}^{\dagger} S_{Q_{2}} \mathcal{U} .
\end{aligned}
$$

The $Q_{1} Q_{1} Q_{2}$ states have normalization $\overline{\mathcal{U}} \mathcal{U} \operatorname{Tr}(\Gamma \bar{\Gamma})$, while for $Q Q Q$ the normalization is $\overline{\mathcal{U}} \mathcal{U} \operatorname{Tr}(\Gamma \bar{\Gamma})+2 \overline{\mathcal{U}} \Gamma \bar{\Gamma} \mathcal{U}$ (which can be understood by counting quark contractions). We define $\bar{\Gamma}=\gamma^{0} \Gamma^{\dagger} \gamma^{0}$ as usual and our spinors satisfy $\bar{u} u=2 m, \bar{u}^{\mu} u_{\mu}=-2 m$ where $m$ is the mass of the state.

We construct amplitudes for semileptonic decays determined by matrix elements of the weak current $j^{\mu}=\bar{c} \gamma^{\mu}\left(1-\gamma_{5}\right) b$. The operator $\bar{c} J^{\mu} b$, where $J^{\mu}=$ $\gamma^{\mu}\left(1-\gamma_{5}\right)$, would be invariant under heavy-quark spin transformations if $J^{\mu}$ transformed as $J^{\mu} \rightarrow S_{c} J^{\mu} S_{b}^{\dagger}$. Thus, we can build matrix elements respecting the heavy-quark spin symmetry by constructing quantities which would be invariant under the same assumption. We observe that $j^{\mu}$ can be rewritten as $j^{\mu}=-\bar{b}^{c} \gamma^{\mu}\left(1+\gamma_{5}\right) c^{c}$ and note that $\bar{b}^{c} J^{c \mu} c^{c}$, where $J^{c \mu}=-\gamma^{\mu}\left(1+\gamma_{5}\right)$, would be invariant if $J^{c \mu} \rightarrow S_{b} J^{c \mu} S_{c}^{\dagger}$.

For the transitions $\Xi_{c c b}^{(*)} \rightarrow \Omega_{c c c}^{*}$, the matrix element respecting heavy-quark symmetry is, up to a scalar function of the product of velocities, $w=v \cdot v^{\prime}$,

$$
\begin{aligned}
& \left\langle\Omega_{c c c}^{*}, v, k, r^{\prime}\left|j^{\mu}(0)\right| \Xi_{c c b}^{(*)}, v, r\right\rangle \\
& =\bar{U}^{\prime}\left(v, k, r^{\prime}\right) J^{\mu} \mathcal{U}(v, r) \operatorname{Tr}\left[\Gamma_{c c b} \bar{\Gamma}_{c c c}\right] \\
& \quad+\overline{\mathcal{U}}^{\prime}\left(v, k, r^{\prime}\right) \Gamma_{c c b} \bar{\Gamma} J^{\mu} \mathcal{U}(v, r),
\end{aligned}
$$

\footnotetext{
${ }^{3}$ Note that the two identical heavy quarks $Q_{1}$ can only be in a symmetric spin- 1 state. The structure

$$
\left[\frac{(1+\not)}{2} \gamma_{\mu} \frac{(1-\not)}{2}\right]
$$

guarantees that the spin of the first two heavy quarks is coupled to 1 (see, for instance, Refs. [21,22]). On the other hand, the spin-1/2 spinor $\left[\frac{1}{\sqrt{3}}\left(v^{\mu}+\gamma^{\mu}\right) \gamma_{5} u(v, r)\right]$ is discussed in Ref. [32].
} 
where $r$ and $r^{\prime}$ are the helicities of the initial and final states, and we use the standard relativistic normalization for hadronic states. Terms with a factor of $\not{v}$ can be omitted because of the equations of motion $(\not u=u, \not \Gamma=\Gamma$, $\gamma_{\mu} u^{\mu}=0, v_{\mu} u^{\mu}=0$ ), while terms with $k$ will always lead to contributions proportional to $v \cdot k$ which is set to 0 at the order we are working. We also make use of the exact relation $\bar{u}^{\prime} k u=0$ and the approximate ones $\bar{u}^{\prime} \gamma_{j} u=$ $\bar{u}^{\prime} v_{j} u, \bar{u}^{\prime} \gamma_{5} u=0$, and $\bar{u}^{\prime} k \gamma_{\mu} \gamma_{5} u=-\bar{u}^{\prime} k v_{\mu} \gamma_{5} u$ valid close to zero recoil.

For the transitions $\Xi_{b b c}^{(*)} \rightarrow \Xi_{c c b}^{(*)}$ the matrix element is

$$
\begin{aligned}
& \left\langle\Xi_{c c b}^{(*)}, v, k, r^{\prime}\left|j^{\mu}(0)\right| \Xi_{b b c}^{(*)}, v, r\right\rangle \\
& \quad=\bar{U}^{\prime}\left(v, k, r^{\prime}\right) \Gamma_{b b c} J^{c \mu} \bar{\Gamma}_{c c b} \mathcal{U}(v, r),
\end{aligned}
$$

while for the transitions $\Omega_{b b b}^{*} \rightarrow \Xi_{b b c}^{(*)}$, the matrix element respecting heavy-quark symmetry now reads

$$
\begin{aligned}
\left\langle\Xi_{b b c}^{(*)}, v, k, r^{\prime}\left|j^{\mu}(0)\right| \Omega_{b b b}^{*}, v, r\right\rangle & \\
= & \bar{U}^{\prime}\left(v, k, r^{\prime}\right) J^{\mu} \mathcal{U}(v, r) \operatorname{Tr}\left[\Gamma_{b b b} \bar{\Gamma}_{b b c}\right] \\
& +\bar{U}^{\prime}\left(v, k, r^{\prime}\right) \Gamma_{b b b} \bar{\Gamma}_{b b c} J^{\mu} \mathcal{U}(v, r) .
\end{aligned}
$$

Close to zero recoil, and within the approximations mentioned above, our results for the transition matrix elements, apart from irrelevant global phases, are

$$
\begin{gathered}
\Xi_{c c b} \rightarrow \Omega_{c c c}^{*}, \quad 2 \eta \bar{u}^{\prime \mu} u, \\
\Xi_{c c b}^{*} \rightarrow \Omega_{c c c}^{*}, \quad-\sqrt{3} \eta \bar{u}^{\prime \lambda} \gamma^{\mu}\left(1-\gamma_{5}\right) u_{\lambda}, \\
\Xi_{b b c} \rightarrow \Xi_{c c b}, \quad-\chi \bar{u}^{\prime}\left(\gamma^{\mu}-\frac{5}{3} \gamma^{\mu} \gamma_{5}\right) u, \\
\Xi_{b b c} \rightarrow \Xi_{c c b}^{*}, \quad-\frac{2}{\sqrt{3}} \chi \bar{u}^{\prime \mu} u, \\
\Xi_{b b c}^{*} \rightarrow \Xi_{c c b}, \quad-\frac{2}{\sqrt{3}} \chi \bar{u}^{\prime} u^{\mu}, \\
\Xi_{b b c}^{*} \rightarrow \Xi_{c c b}^{*}, \quad-2 \chi \bar{u}^{\prime \lambda} \gamma^{\mu}\left(1-\gamma_{5}\right) u_{\lambda}, \\
\Omega_{b b b}^{*} \rightarrow \Xi_{b b c}, \quad 2 \xi \bar{u}^{\prime} u^{\mu}, \\
\Omega_{b b b}^{*} \rightarrow \Xi_{b b c}^{*}, \quad-\sqrt{3} \xi \bar{u}^{\prime \lambda} \gamma^{\mu}\left(1-\gamma_{5}\right) u_{\lambda},
\end{gathered}
$$

where the factors $\eta(w), \chi(w)$, and $\xi(w)$ are the Isgur-Wise functions that depend on $w=v \cdot v^{\prime}$ and that we expect to be close to 1 at zero recoil $(w=1)$. In fact in the limit $m_{c}=m_{b}$ they would be exactly 1 at zero recoil. To check that assertion let us consider an $S U(2)$ symmetry under which the $c$ and $b$ quarks transform as a doublet and the four states $\Omega_{c c c}^{*}, \Xi_{c c b}^{*}, \Xi_{b b c}^{*}$, and $\Omega_{b b b}^{*}$ form a quadruplet. We will consider all heavy-quark spins aligned, that is to say we will place the four baryons in the state with maximum third component of spin, $J_{z}=+3 / 2$. The $\mu=0$ component of the vector part of the transition operator $j^{\mu}$ would then be $I_{+}=c^{\dagger} b$, which is the raising operator in the Fock space for this flavor $S U(2)$ symmetry. Assuming this symmetry, and taking into account the state normalization, we will have at zero recoil

$$
\begin{gathered}
\sqrt{3}=\frac{1}{2 m}\left\langle\Omega_{c c c}^{*}\left|c^{\dagger} b\right| \Xi_{c c b}^{*}\right\rangle=\sqrt{3} \eta(1), \\
2=\frac{1}{2 m}\left\langle\Xi_{c c b}^{*}\left|c^{\dagger} b\right| \Xi_{b b c}^{*}\right\rangle=2 \chi(1), \\
\sqrt{3}=\frac{1}{2 m}\left\langle\Xi_{b b c}^{*}\left|c^{\dagger} b\right| \Omega_{b b b}^{*}\right\rangle=\sqrt{3} \xi(1) .
\end{gathered}
$$

In the above equations, the leftmost results follow from the $c \leftrightarrow b S U(2)$ symmetry, while the rightmost ones are obtained from Eqs. (10), (14), and (16). From Eqs. (17)-(19), we deduce $\eta(1)=\chi(1)=\xi(1)=1$. For the actual quark masses one expects deviations from this result as a consequence of a mismatch between the initial and final wave functions.

\section{DECAY WIDTH FOR A SEMILEPTONIC $b \rightarrow c$ TRANSITION AND HQSS CONSTRAINTS}

The total decay width for semileptonic $b \rightarrow c$ baryon transitions is given by

$\Gamma=\left|V_{c b}\right|^{2} \frac{G_{F}^{2}}{8 \pi^{4}} \frac{m^{\prime 2}}{m} \int \sqrt{w^{2}-1} \mathcal{L}^{\alpha \beta}(q) \mathcal{H}_{\alpha \beta}(v, k) d w$,

where $\left|V_{c b}\right|$ is the modulus of the corresponding CabibboKobayashi-Maskawa matrix element for a $b \rightarrow c$ quark transition, for which we shall use $\left|V_{b c}\right|=0.0410$ [33]. $G_{F}=1.16637(1) \times 10^{-11} \mathrm{MeV}^{-2}$ [33] is the Fermi decay constant and $q=p-p^{\prime} . w$ and $q^{2}$ are related by $w=$ $\frac{m^{2}+m^{\prime 2}-q^{2}}{2 m m^{\prime}}$. In the decay, $w$ ranges from $w=1$, corresponding to zero recoil of the final baryon, to a maximum value given, neglecting the neutrino mass, by $w=w_{\max }=$ $\frac{m^{2}+m^{2}-m_{l}^{2}}{2 m m^{\prime}}$, where $m_{l}$ is the final charged lepton mass. Finally $\mathcal{L}^{\alpha \beta}(q)$ is the leptonic tensor after integrating over the lepton momenta and $\mathcal{H}_{\alpha \beta}(v, k)$ is the hadronic tensor.

The leptonic tensor is given by

$$
\mathcal{L}^{\alpha \beta}(q)=A\left(q^{2}\right) g^{\alpha \beta}+B\left(q^{2}\right) \frac{q^{\alpha} q^{\beta}}{q^{2}},
$$

where

$$
\begin{aligned}
& A\left(q^{2}\right)=-\frac{I\left(q^{2}\right)}{6}\left(2 q^{2}-m_{l}^{2}-\frac{m_{l}^{4}}{q^{2}}\right), \\
& B\left(q^{2}\right)=\frac{I\left(q^{2}\right)}{3}\left(q^{2}+m_{l}^{2}-2 \frac{m_{l}^{4}}{q^{2}}\right)
\end{aligned}
$$

with 


$$
I\left(q^{2}\right)=\frac{\pi}{2 q^{2}}\left(q^{2}-m_{l}^{2}\right)
$$

The hadronic tensor reads

$$
\begin{aligned}
\mathcal{H}^{\alpha \beta}(v, k)= & \frac{1}{2 J+1} \sum_{r, r^{\prime}}\left\langle B^{\prime}, v, k, r^{\prime}\left|j^{\alpha}(0)\right| B, v, r\right\rangle \\
& \times\left\langle B^{\prime}, v, k, r^{\prime}\left|j^{\beta}(0)\right| B, v, r\right\rangle^{*}
\end{aligned}
$$

with $J$ the initial baryon spin. Baryonic states are normalized such that

$$
\left\langle B, v^{\prime}, r^{\prime} \mid B, v, r\right\rangle=2 E(2 \pi)^{3} \delta_{r r^{\prime}} \delta\left(\vec{p}-\vec{p}^{\prime}\right)
$$

with $E$ the baryon energy for three-momentum $\vec{p}$.

\section{HQSS constraints on semileptonic decay widths}

For large quark masses and near zero recoil we can use the HQSS results in Eqs. (9)-(16) to approximate the product $\mathcal{L}^{\alpha \beta} \mathcal{H}_{\alpha \beta}$ by

(i) $c c b \rightarrow c c c$ transitions:

$\Xi_{c c b} \rightarrow \Omega_{c c c}^{*}$

$$
\begin{aligned}
\mathcal{L}^{\alpha \beta} \mathcal{H}_{\alpha \beta} \approx & \frac{16}{3} \eta^{2} m m^{\prime}(1+w)\left[-3 A\left(q^{2}\right)\right. \\
& \left.+B\left(q^{2}\right)\left(\frac{\left(v^{\prime} \cdot q\right)^{2}}{q^{2}}-1\right)\right] \\
\Xi_{c c b}^{*} \rightarrow \Omega_{c c c}^{*} & \\
\mathcal{L}^{\alpha \beta} \mathcal{H}_{\alpha \beta} \approx & \frac{1}{3} \eta^{2} m m^{\prime}\left[-8 A\left(q^{2}\right) w\left(1+2 w^{2}\right)\right. \\
& +B\left(q^{2}\right)\left(-w\left(12+8 w^{2}\right)\right. \\
& \left.\left.+2 \frac{(v \cdot q)\left(v^{\prime} \cdot q\right)}{q^{2}}\left(20+8 w^{2}\right)\right)\right]
\end{aligned}
$$

(ii) $b b c \rightarrow c c b$ transitions:

$$
\begin{aligned}
\Xi_{b b c} \rightarrow \Xi_{c c b} & \\
\mathcal{L}^{\alpha \beta} \mathcal{H}_{\alpha \beta} \approx & \frac{4}{9} \chi^{2} m m^{\prime}\left\{-A\left(q^{2}\right)(34 w+32)\right. \\
& \left.+B\left(q^{2}\right)\left[17\left(2 \frac{(v \cdot q)\left(v^{\prime} \cdot q\right)}{q^{2}}-w\right)-8\right]\right\}
\end{aligned}
$$

$$
\begin{aligned}
\Xi_{b b c}^{*} \rightarrow \Xi_{c c b} & \begin{aligned}
\mathcal{L}^{\alpha \beta} \mathcal{H}_{\alpha \beta} \approx & \frac{8}{9} \chi^{2} m m^{\prime}(1+w)\left[-3 A\left(q^{2}\right)\right. \\
& \left.+B\left(q^{2}\right)\left(\frac{(v \cdot q)^{2}}{q^{2}}-1\right)\right]
\end{aligned}
\end{aligned}
$$

$$
\begin{aligned}
\Xi_{b b c} \rightarrow \Xi_{c c b}^{*} & \\
\mathcal{L}^{\alpha \beta} \mathcal{H}_{\alpha \beta} \approx & \frac{16}{9} \chi^{2} m m^{\prime}(1+w)\left[-3 A\left(q^{2}\right)\right. \\
& \left.+B\left(q^{2}\right)\left(\frac{\left(v^{\prime} \cdot q\right)^{2}}{q^{2}}-1\right)\right] \\
\Xi_{b b c}^{*} \rightarrow \Xi_{c c b}^{*} & \begin{aligned}
\mathcal{L}^{\alpha \beta} \mathcal{H}_{\alpha \beta} \approx & \frac{4}{9} \chi^{2} m m^{\prime}\left[-8 A\left(q^{2}\right) w\left(1+2 w^{2}\right)\right. \\
& +B\left(q^{2}\right)\left(-w\left(12+8 w^{2}\right)\right. \\
& \left.\left.+2 \frac{(v \cdot q)\left(v^{\prime} \cdot q\right)}{q^{2}}\left(20+8 w^{2}\right)\right)\right]
\end{aligned}
\end{aligned}
$$

(iii) $b b b \rightarrow b b c$ transitions:

$$
\begin{aligned}
& \Omega_{b b b}^{*} \rightarrow \Xi_{b b c} \\
& \mathcal{L}^{\alpha \beta} \mathcal{H}_{\alpha \beta} \approx \frac{8}{3} \xi^{2} m m^{\prime}(1+w)\left[-3 A\left(q^{2}\right)\right. \\
& \left.+B\left(q^{2}\right)\left(\frac{(v \cdot q)^{2}}{q^{2}}-1\right)\right] \\
& \Omega_{b b b}^{*} \rightarrow \Xi_{b b c}^{*} \\
& \mathcal{L}^{\alpha \beta} \mathcal{H}_{\alpha \beta} \approx \frac{1}{3} \xi^{2} m m^{\prime}\left[-8 A\left(q^{2}\right) w\left(1+2 w^{2}\right)\right. \\
& +B\left(q^{2}\right)\left(-w\left(12+8 w^{2}\right)\right. \\
& \left.\left.+2 \frac{(v \cdot q)\left(v^{\prime} \cdot q\right)}{q^{2}}\left(20+8 w^{2}\right)\right)\right]
\end{aligned}
$$

In the strict near-zero-recoil approximation, $\omega \approx 1$ or equivalently $q^{2}$ very close to its maximum value $q_{\max }^{2}$, we can approximate

$$
\frac{(v \cdot q)^{2}}{q^{2}} \approx \frac{\left(v^{\prime} \cdot q\right)(v \cdot q)}{q^{2}} \approx \frac{\left(v^{\prime} \cdot q\right)^{2}}{q^{2}} \approx 1 .
$$

In addition, $A\left(q^{2}\right) \approx-B\left(q^{2}\right)$ near $q_{\max }^{2}$. To the extent that the former approximations are good and further using

$$
m_{B_{b b c}} \approx m_{B_{b b c}^{*}}, \quad m_{B_{c c b}} \approx m_{B_{c c b}^{*}},
$$

we can make approximate, but model independent, predictions for ratios of semileptonic $b \rightarrow c$ decay widths based in the above HQSS relations for $\mathcal{L}^{\alpha \beta} \mathcal{H}_{\alpha \beta}$. We find

$$
\begin{gathered}
\frac{\Gamma\left(\Xi_{c c b} \rightarrow \Omega_{c c c}^{*}\right)}{\frac{8}{5} \Gamma\left(\Xi_{c c b}^{*} \rightarrow \Omega_{c c c}^{*}\right)} \approx 1, \\
\frac{\Gamma\left(\Xi_{b b c} \rightarrow \Xi_{c c b}\right)}{\frac{25}{8} \Gamma\left(\Xi_{b b c} \rightarrow \Xi_{c c b}^{*}\right)} \approx 1,
\end{gathered}
$$


TABLE I. Masses (in MeV) of the triply heavy baryons obtained with the AL1 potential of Refs. [3,34] using our variational approach. For comparison we also show the results from the Faddeev calculation performed in Ref. [3] using the same potential. Predicted masses within other theoretical approaches are also compiled. Hyperfine splitting is neglected in [11].

\begin{tabular}{|c|c|c|c|c|c|c|c|c|c|c|c|}
\hline & $\begin{array}{l}\text { This work } \\
\text { Variational }\end{array}$ & $\begin{array}{c}{[3]} \\
\text { Faddeev }\end{array}$ & $\begin{array}{c}{[13]} \\
\text { LQCD }\end{array}$ & $\begin{array}{l}{[1]} \\
\mathrm{BM}\end{array}$ & [2] & $\begin{array}{c}{[4]} \\
\text { NRCQM }\end{array}$ & $\begin{array}{c}{[11]} \\
\text { LO pNRQCD }\end{array}$ & $\begin{array}{c}{[5]} \\
\text { NRCQM }\end{array}$ & $\begin{array}{c}{[6]} \\
\text { RTQM }\end{array}$ & $\begin{array}{c}{[7]} \\
\text { Regge }\end{array}$ & $\begin{array}{c}{[10]} \\
\text { QCDSR }\end{array}$ \\
\hline & 14398 & 14398 & $14371 \pm 12$ & 14300 & 14760 & $\ldots$ & $14370 \pm 80$ & 14834 & 14569 & $\ldots$ & $13280 \pm 100$ \\
\hline$\Xi$ & 11245 & $\ldots$ & $\ldots$ & 11200 & $11480 \pm 120$ & $\ldots$ & $11190 \pm 80$ & 11554 & 11287 & $\ldots$ & $10540 \pm 110$ \\
\hline$m_{\Xi_{b b c}}$ & 11214 & 11217 & $\cdots$ & $\cdots$ & $\cdots$ & $\cdots$ & $11190 \pm 80$ & 11535 & 11280 & $\cdots$ & $10300 \pm 100$ \\
\hline$n_{\Xi^{*}}^{*}$ & 8046 & & $\cdots$ & 8030 & $8200 \pm 90$ & $\cdots$ & $7980 \pm 70$ & 8265 & 8025 & $\cdots$ & $7450 \pm 160$ \\
\hline$n_{\Xi}=c b$ & 8018 & 8019 & $\ldots$ & $\ldots$ & $\ldots$ & $\ldots$ & $7980 \pm 70$ & 8245 & 8018 & $\ldots$ & $7410 \pm 130$ \\
\hline$m_{\Omega_{c c c}^{*}}^{-c t}$ & 4799 & 4799 & $\cdots$ & 4790 & $4925 \pm 90$ & 4632 & $4760 \pm 60$ & 4965 & 4803 & $4819 \pm 7$ & $4670 \pm 150$ \\
\hline
\end{tabular}

$$
\begin{aligned}
& \frac{2 \Gamma\left(\Xi_{b b c}^{*} \rightarrow \Xi_{c c b}\right)}{\Gamma\left(\Xi_{b b c} \rightarrow \Xi_{c c b}^{*}\right)} \approx 1, \\
& \frac{\Gamma\left(\Xi_{b b c}^{*} \rightarrow \Xi_{c c b}^{*}\right)}{\frac{5}{2} \Gamma\left(\Xi_{b b c} \rightarrow \Xi_{c c b}^{*}\right)} \approx 1, \\
& \frac{\Gamma\left(\Omega_{b b b}^{*} \rightarrow \Xi_{b b c}\right)}{\frac{4}{5} \Gamma\left(\Omega_{b b b}^{*} \rightarrow \Xi_{b b c}^{*}\right)} \approx 1 .
\end{aligned}
$$

These relations are similar to the ones we obtained in our former study of doubly heavy baryons [27] and from the findings of this latter work, we expect them to hold at the level of $20 \%$. To estimate the decay widths themselves, we need to know the Isgur-Wise functions $\eta(w), \chi(w)$, and $\xi(w)$. In the next section we will use a nonrelativistic constituent quark model for this purpose.

\section{NONRELATIVISTIC QUARK MODEL EVALUATION OF THE ISGUR-WISE FUNCTIONS AND DECAY WIDTHS}

In this section we shall obtain, within the nonrelativistic quark model and using the AL1 interquark potential of Refs. [3,34], the wave functions of the heavy baryons involved in this study. With those wave functions we can evaluate the Isgur-Wise functions and estimate the baryon semileptonic $b \rightarrow c$ decay widths.

The wave functions have the general form

$$
\begin{aligned}
\Psi_{\alpha_{1} \alpha_{2} \alpha_{3}}= & \delta_{f_{1} h} \delta_{f_{2} h} \delta_{f_{3} h^{\prime}} \frac{\epsilon_{c_{1} c_{2} c_{3}}}{\sqrt{3 !}} \Phi\left(r_{1}, r_{2}, r_{12}\right) \\
& \times\left(1 / 2,1 / 2,1 ; s_{1}, s_{2}, s_{1}+s_{2}\right) \\
& \times\left(1,1 / 2, J ; s_{1}+s_{2}, s_{3}, M\right),
\end{aligned}
$$

where $\alpha_{j}$ represents the spin $(s)$, flavor $(f)$, and color $(c)$ quantum numbers of the $j$ th quark. The two first quarks have the same flavor $h$, while the third quark has flavor $h^{\prime}$, which could be also the same as the one of the first two. $\epsilon_{c_{1} c_{2} c_{3}} / \sqrt{3}$ ! is the fully antisymmetric color wave function and the $\left(j_{1}, j_{2}, j ; m_{1}, m_{2}, m\right)$ are $S U(2)$ Clebsch-Gordan coefficients. $J$ is the total spin of the baryon. As we are interested only in spin- $1 / 2$ or $-3 / 2$ ground-state baryons, the total orbital angular momentum is $L=0$. Thus, the orbital part of the wave function can only depend on the modulus of the relative distances between the quarks. Here we use $r_{1}, r_{2}$ which are the relative distances between quark three and quarks one and two, respectively, and $r_{12}$ which is the relative distance between the first two quarks. Following our works on single and double heavy baryons $[24,25]$ we shall use a variational ansatz to solve the threebody problem. We write the orbital wave functions as the product of three functions, each one depending on just one of the three variables $r_{1}, r_{2}, r_{12}$; i.e.,

$$
\Phi\left(r_{1}, r_{2}, r_{12}\right)=\phi_{h h^{\prime}}\left(r_{1}\right) \phi_{h h^{\prime}}\left(r_{2}\right) \phi_{h h}\left(r_{12}\right) .
$$

For each of the $\phi$ functions above we take an expression of the form ${ }^{4}$

$$
\phi(r)=\sum_{j=1}^{4} a_{j} e^{-b_{j}^{2}\left(r+d_{j}\right)^{2}} \quad\left(a_{1}=1\right) .
$$

The variational parameters are fixed by minimizing the energy and the overall normalization is fixed at the end of the calculation. The results we get for the masses are given in Table I, where we also compare them to the ones obtained in Ref. [3] using the same potential but solving Faddeev equations. The agreement between the two approaches is very good.

There is a recent estimate [13] of the mass of the triply heavy baryon $b b b$ obtained in lattice QCD with $2+1$ flavors of light sea quarks. Our result compares with it rather well. Our predictions are also in a reasonable agreement with those obtained within the BM, RTQM, and LO pNRQCD evaluations of Refs. [1,6,11], respectively. The QCDSR masses calculated in [10] come out systematically much smaller than ours, while those obtained in the NRCQM of Ref. [5] are significantly larger than our predictions.

\footnotetext{
${ }^{4}$ We use four Gaussians in the present approach. We have checked that by increasing the number of Gaussians, the variational baryon masses change in less than $5 \mathrm{MeV}$.
} 
Before computing the Isgur-Wise functions that govern the semileptonic decays of the triply heavy baryons, we would like to devote a few words to discussing the confinement potential in these systems. In phenomenological constituent quark models, such as the AL1 potential used here, the confinement potential for baryons is usually obtained from the two-body forces that describe the dynamics of each quark pair. However, the lattice QCD simulations carried out in Refs. [35,36] seem to indicate that, in a three static quark system, confinement is a genuine three-body effect. Changes in the masses due to the use of one or other of these approaches are studied in the next subsection.

\section{A. $\Delta$-shaped versus $Y$-shaped potential}

LQCD results for the static quark-antiquark ground-state potential [37] are well described by a dependence

$$
-\frac{A}{r}+\sigma r+C
$$

which contains the sum of the short-distance Coulomb onegluon exchange (OGE) term plus the confining longdistance flux-tube contribution. Most phenomenological models assume such a dependence and fit the $A, \sigma$, and $C$ parameters to the meson spectrum. This is, for instance, the case of the AL1 potential that we use. When going to the quark-quark sector, a factor of $1 / 2$, assumed to come from an overall color $\vec{\lambda} \cdot \vec{\lambda}$ dependence, ${ }^{5}$ is added to the interaction. The resulting potential in baryons is thus obtained as the sum of two-body terms. For the confining part, one is summing over the three sides of a triangle with the quarks located at its vertices $\left[\sigma\left(r_{1}+r_{2}+r_{12}\right) / 2\right]$, leading to the name of $\Delta$-shaped potential (see Fig. 1). This picture works very well from a phenomenological point of view and one gets a good description of the light and single heavy baryon spectrum once the parameters have been fixed in the corresponding meson sector.

As mentioned above, the 3 quark static potential has been directly measured on the lattice in Refs. [35,36]. A good fit to the lattice data was obtained assuming a picture, similar to the one described above, in which the potential has a short-distance Coulomb OGE part plus a longdistance flux-tube part

$$
-A_{3 q}\left(\frac{1}{r_{1}}+\frac{1}{r_{2}}+\frac{1}{r_{12}}\right)+\sigma_{3 q} L_{\min }+C_{3 q}
$$

where $L_{\min }$ is the minimal value of the total length of the color flux tubes linking the three quarks. The flux tubes adopt a $Y$ shape (See Fig. 1), hence the name $Y$-shaped potential. This is in agreement with the picture that emerges from the QCD BM calculations carried out in Ref. [1]. Indeed the $\Delta$ and $Y$ nomenclature was already

\footnotetext{
${ }^{5}$ QCD predicts exactly this color factor for the OGE term.
}

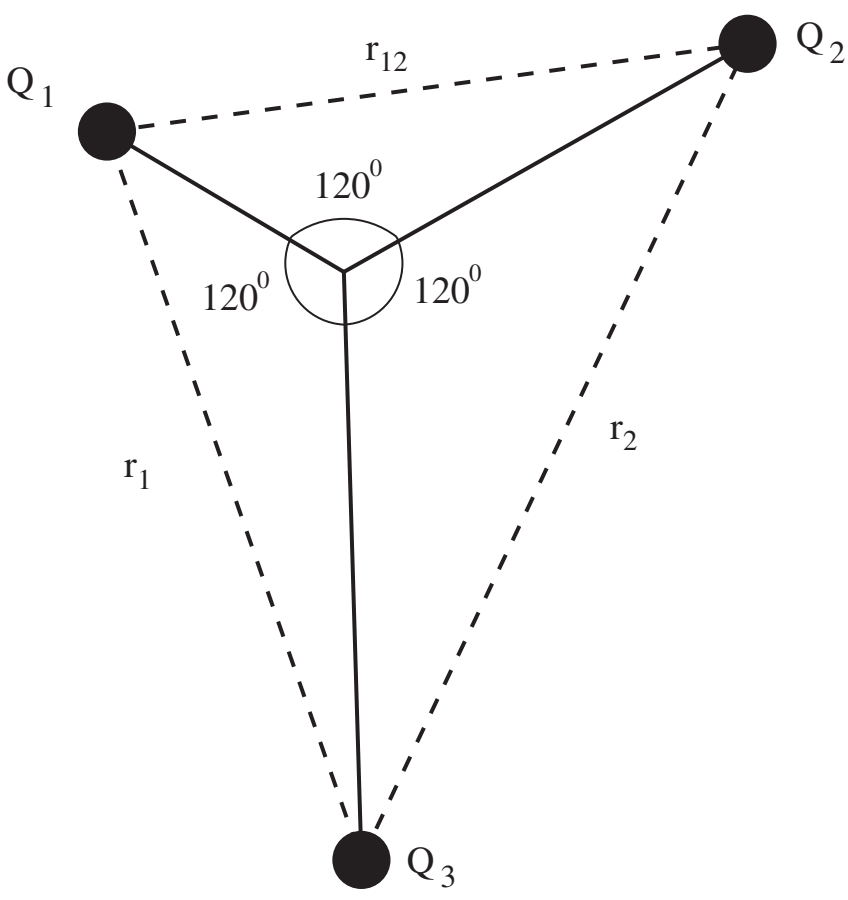

FIG. 1. Illustration of the $\Delta$ - and $Y$-shaped confinement potentials.

used in this pioneering work of 1980. In terms of the $r_{1}, r_{2}$, and $r_{12}$ interquark distances one has

$$
L_{\min }^{2}=\frac{1}{2}\left(r_{1}^{2}+r_{2}^{2}+r_{12}^{2}\right)+\frac{\sqrt{3}}{2} \sqrt{-\lambda\left(r_{1}^{2}, r_{2}^{2}, r_{12}^{2}\right)}
$$

when none of the angles of the three quark triangle exceeds $2 \pi / 3$ and where $\lambda(a, b, c)=a^{2}+b^{2}+c^{2}-2 a b-$ $2 a c-2 b c$. If one of the triangle's angles exceeds $2 \pi / 3$ then $L_{\min }$ is just given by

$$
L_{\min }=r_{1}+r_{2}+r_{12}-\max \left(r_{1}, r_{2}, r_{12}\right) .
$$

Comparing this fit with the one for the quark-antiquark potential they found that $\sigma_{3 q} \approx \sigma, A_{3 q} \approx \frac{1}{2} A$, and $C_{3 q} \approx$ $\frac{3}{2} C$. Thus, leaving out the confinement piece, one could approximate the 3 quark potential by the sum of three twobody quark-quark terms. For the confining part, the sum of three two-body quark-quark terms is always smaller than the three-body force obtained from lattice QCD data. Actually, one has $\left(r_{1}+r_{2}+r_{12}\right) / 2 \leq L_{\min } \leq\left(r_{1}+r_{2}+\right.$ $\left.r_{12}\right) / \sqrt{3}$, which might induce changes of around $15 \%$ at most in this part of the potential. Indeed, in Ref. [35] the lattice data were also fitted to the sum of the three quarkquark potentials ${ }^{6}$ and it was found that a slightly larger value for the confinement coefficient $(0.53 \sigma$ vs $\sigma / 2)$ was required. The ad hoc factor of $1 / 2$ introduced in quark potentials when going from the mesons to baryons is

\footnotetext{
${ }^{6}$ The fit is worse than that obtained when the functional form of Eq. (45) is used.
} 
TABLE II. First column: Masses (in MeV) of the triply heavy baryons obtained with the AL1 potential of Refs. [3,34] using our variational approach. Second column: The same by substituting $\sigma\left(r_{1}+r_{2}+r_{12}\right) / 2$ by $\sigma L_{\min }$ in the AL1 potential.

This work

\begin{tabular}{lcc} 
& $\Delta$-shaped potential & $Y$-shaped potential \\
\hline$m_{\Omega_{b b b}^{*}}$ & 14398 & 14424 \\
$m_{\Xi_{b b c}^{*}}$ & 11245 & 11281 \\
$m_{\Xi_{b b c}}$ & 11214 & 11247 \\
$m_{\Xi_{c c b}^{*}}$ & 8046 & 8087 \\
$m_{\Xi_{c c b}}$ & 8018 & 8058 \\
$m_{\Omega_{c c c}^{*}}$ & 4799 & 4847 \\
\hline \hline
\end{tabular}

understood here as a geometrical effect rather than as a color factor as it is usually presented.

To be more quantitative, we have computed the triply heavy baryon masses also with a $Y$-shaped confinement potential. To that end, we have taken the AL1 potential used before, and have replaced the $\sigma\left(r_{1}+r_{2}+r_{12}\right) / 2$ term by $\sigma L_{\text {min }}$. Results are presented in Table II. There we also compare with the masses obtained previously using the AL1 potential. We see a small increase in the masses of roughly $26,34,40$, and $48 \mathrm{MeV}$ for the $b b b$, $b b c, c c b$, and $c c c$ systems, respectively. Effects here are similar to those due to the hyperfine splitting. Future and precise measurements of the masses might help to shed light on the exact nature of the confinement potential in the baryon sector.

The corrections to the ratios of decay widths, which would be computed in the subsections below, are even smaller, as expected from perturbation theory, since changes in the wave functions arise at second order.

\section{B. Isgur-Wise functions}

To evaluate the Isgur-Wise functions we follow our work in Ref. [26] and write ${ }^{7}$

$$
\begin{aligned}
\eta_{\Xi^{(*)}}(|\vec{q}|)= & \int d^{3} r_{1} d^{3} r_{2} e^{i \vec{q} \cdot\left(m_{c} \vec{r}_{1}+m_{c} \vec{r}_{2}\right) / \bar{M}_{c c c}} \\
& \times\left[\Psi_{\Omega_{c c c}^{*}}\left(r_{1}, r_{2}, r_{12}\right)\right]^{*} \Psi_{\Xi_{c c b}^{(*)}}\left(r_{1}, r_{2}, r_{12}\right), \\
\chi_{\Xi^{(*)} \rightarrow \Xi, \Xi^{*}}(|\vec{q}|)= & \int d^{3} r_{1} d^{3} r_{2} e^{-i \vec{q} \cdot\left(m_{b} \vec{r}_{12}+m_{c} \vec{r}_{1}\right) / \bar{M}_{c c b}} \\
& \times\left[\Psi_{\Xi_{c c b}^{(*)}}\left(r_{12}, r_{2}, r_{1}\right)\right]^{*} \Psi_{\Xi_{b b c}^{(*)}}\left(r_{1}, r_{2}, r_{12}\right),
\end{aligned}
$$

$$
\begin{aligned}
\xi_{\Xi^{(*)}}(|\vec{q}|)= & \int d^{3} r_{1} d^{3} r_{2} e^{i \vec{q} \cdot\left(m_{b} \vec{r}_{1}+m_{b} \vec{r}_{2}\right) / \bar{M}_{b b c}} \\
& \times\left[\Psi_{\Xi_{b b c}^{(*)}}\left(r_{1}, r_{2}, r_{12}\right)\right]^{*} \Psi_{\Omega_{b b b}^{*}}\left(r_{1}, r_{2}, r_{12}\right),
\end{aligned}
$$

\footnotetext{
${ }^{7}$ Note when the initial baryon is at rest, $w=\frac{E^{\prime}}{m^{\prime}}$ is just a function of $|\vec{q}|$.
}

where $\bar{M}_{h h h^{\prime}}=m_{h}+m_{h}+m_{h^{\prime}}$. In Fig. 2, we display the eight overlap functions obtained from each of the decays examined here. We see that as predicted by HQSS in Eqs. (9)-(16) they reduce to only three independent ones in very good approximation. In the equal-mass case they would be equal to one at zero recoil $(|\vec{q}|=0)$. For finite masses we see they deviate slightly from 1 at zero recoil owing to the mismatch between the initial and final wave functions. Note that $w=1+v \cdot k / m^{\prime}$, and thus as $w$ departs from $1, v \cdot k / m^{\prime}$ increases. To obtain the relations of Eqs. (9)-(16) all $v \cdot \mathrm{k} / \mathrm{m}^{\prime}$ corrections were neglected. Thus, the Isgur-Wise (overlap) functions depicted in Fig. 2 would provide a poorer description of the weak transition matrix elements as $w$ deviates from the zero-recoil point. The largest corrections are expected for the $b c c \rightarrow c c c$ transitions, related to the $\eta$-type Isgur-Wise functions in Fig. 2 , for which $w_{\max } \approx 1.125$. For this case at the $q^{2}=0$ end of the phase space, $v \cdot k / m^{\prime}$ becomes of order $1 / 8$. Thus, in this region, approximating the full amplitude (weak matrix element) by means of the $\eta(w)$ Isgur-Wise function could be subject to uncertainties of order of $15 \%-25 \%$. For $b b c \rightarrow b c c$ or $b b b \rightarrow b b c$ transitions, the $\chi-$ and $\xi-$ Isgur-Wise functions should provide more accurate estimates of the transition weak matrix elements for the whole available phase space, since in those cases $w_{\max }$ is only about 1.06 and 1.03 , respectively.

In the next subsection, we will make use of the IsgurWise functions of Fig. 2 to estimate the decay widths. This should be quite accurate, even for the $b c c \rightarrow c c c$ transitions, since, as we will see, the differential decay width distribution peaks very close to the zero-recoil point and hence far from the end point of the spectrum $w=w_{\max }$. Indeed, for $b c c \rightarrow c c c$ transitions, the distribution takes its

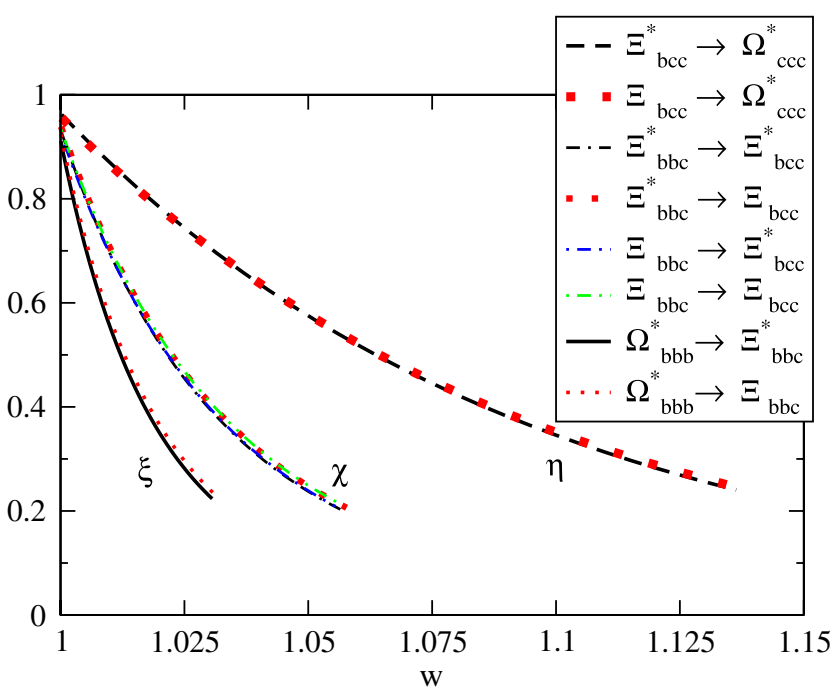

FIG. 2 (color online). Overlap functions for $b \rightarrow c$ semileptonic decays of triply heavy baryons obtained in a nonrelativistic quark model. The functions fall into three families, consistent with heavy-quark spin symmetry. 

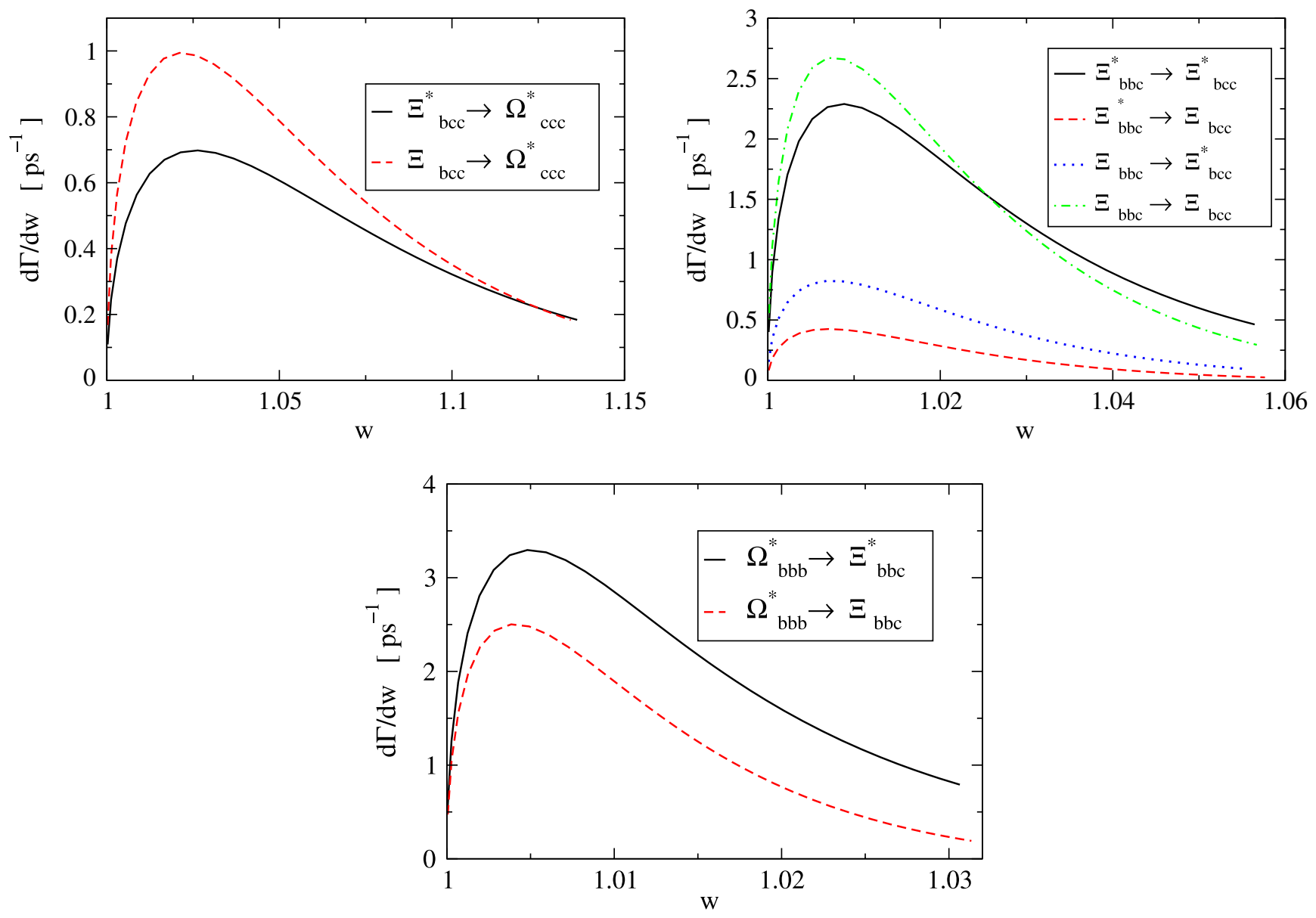

FIG. 3 (color online). Estimated $\frac{d \Gamma}{d w}$ differential decay widths in $\mathrm{ps}^{-1}$ for the different transitions considered.

maximum value well below $w=1.05$ (see left upper panel of Fig. 3).

\section{Decay widths}

We now use the HQSS approximate expressions in Eqs. (26)-(33) to estimate the decay widths. The results for the differential distributions are shown in Fig. 3 and the integrated decay widths are compiled in Table III. We see the ratios in Eqs. (37) and (38) are satisfied at the level of $5.5 \%$ and $3.4 \%$, respectively, whereas the ratios in Eqs. (36), (39), and (40) are good only at the level of $20 \%-30 \%$. It is clear the relations in Eqs. (36)-(40) can only be approximate. First, the strict zero-recoil point is forbidden by phase space, and second, $q^{2}$ changes rapidly from its maximum value of $\left(m-m^{\prime}\right)^{2}$ at $w=1$ to its minimum value of $m_{l}^{2}$ at $w_{\max }$ which makes the approximation in Eq. (34) not good enough. ${ }^{8}$

\footnotetext{
${ }^{8}$ Note, as pointed out in Ref. [27], the quantities $(v \cdot q)^{2} / q^{2}$, $\left(v^{\prime} \cdot q\right)(v \cdot q) / q^{2}$, and $\left(v^{\prime} \cdot q\right)^{2} / q^{2}$ which are all equal to 1 near zero recoil, quickly deviate from 1 because of the $q^{2}$ factor in the denominator.
}

What one sees when looking at the differential decay widths in Fig. 3 is that these distributions peak in each case in the lower part of the allowed $w$ region, about 1.005, 1.009 , and 1.025 for $b b b, b b c$, and $c c b$ decays, respectively, quite close to the zero-recoil point. In these circumstances one can relax the strict approximation in Eq. (34) and use instead [27]

TABLE III. Estimated decay widths in units of $\mathrm{ps}^{-1}$. We use $\left|V_{b c}\right|=0.0410$. Similar results are obtained for $\mu \bar{\nu}_{\mu}$ leptons in the final state.

\begin{tabular}{lc}
\hline \hline$B \rightarrow B^{\prime} e \bar{\nu}_{e}$ & $\Gamma\left[\mathrm{ps}^{-1}\right]$ \\
\hline$\Xi_{c c b} \rightarrow \Omega_{c c c}^{*} e \bar{\nu}_{e}$ & $8.01 \times 10^{-2}$ \\
$\Xi_{c c b}^{*} \rightarrow \Omega_{c c c}^{*} e \bar{\nu}_{e}$ & $6.28 \times 10^{-2}$ \\
$\Xi_{b b c} \rightarrow \Xi_{c c b} e \bar{\nu}_{e}$ & $7.98 \times 10^{-2}$ \\
$\Xi_{b b c} \rightarrow \Xi_{c c b}^{*} e \bar{\nu}_{e}$ & $2.42 \times 10^{-2}$ \\
$\Xi_{b b c}^{*} \rightarrow \Xi_{c c b} e \bar{\nu}_{e}$ & $1.17 \times 10^{-2}$ \\
$\Xi_{b b c}^{*} \rightarrow \Xi_{c c b}^{*} e \bar{\nu}_{e}$ & $7.74 \times 10^{-2}$ \\
$\Omega_{b b b}^{*} \rightarrow \Xi_{b b c}^{*} e \bar{\nu}_{e}$ & $3.95 \times 10^{-2}$ \\
$\Omega_{b b b}^{*} \rightarrow \Xi_{b b c}^{*} e \bar{\nu}_{e}$ & $6.34 \times 10^{-2}$ \\
\hline \hline
\end{tabular}




$$
\frac{(v \cdot q)^{2}}{q^{2}} \approx \frac{(v \cdot q)\left(v^{\prime} \cdot q\right)}{q^{2}} \approx \frac{\left(v^{\prime} \cdot q\right)^{2}}{q^{2}}
$$

which should be reasonable near the maximum of the differential decay width, since we can still use $w \approx 1$. We can also use $B\left(q^{2}\right) \approx-A\left(q^{2}\right)$ and the approximate equality of masses in Eq. (35). One can now only make the following two model independent predictions,

$$
\begin{gathered}
\frac{2 \Gamma\left(\Xi_{b b c}^{*} \rightarrow \Xi_{c c b}\right)}{\Gamma\left(\Xi_{b b c} \rightarrow \Xi_{c c b}^{*}\right)} \approx 1, \\
\frac{\Gamma\left(\Xi_{b b c}^{*} \rightarrow \Xi_{c c b}^{*}\right)}{4 \Gamma\left(\Xi_{b b c} \rightarrow \Xi_{c c b}\right)-10 \Gamma\left(\Xi_{b b c} \rightarrow \Xi_{c c b}^{*}\right)} \approx 1,
\end{gathered}
$$

which we see are good at the level of $3.4 \%$ and $0.25 \%$, respectively.

\section{CONCLUSIONS}

We have studied the $b \rightarrow c$ semileptonic decays of the lowest-lying triply heavy $\left(Q_{1} Q_{2} Q_{3}\right.$, with $\left.Q_{i}=b, c\right)$ baryons in the limit $m_{b}, m_{c} \gg \Lambda_{\mathrm{QCD}}$ and close to the zerorecoil point. The separate heavy-quark spin symmetries strongly constrain the matrix elements, leading to single form factors for all these decays. We have obtained these HQSS relations for the first time. Lattice QCD simulations work best near the zero-recoil point and thus are well suited to check the validity of our results.

We have used a NRCQM, adjusted to the meson spectrum, to predict the masses of these triply heavy baryons by using a simple variational scheme. Results for masses compare rather well with some previous Faddeev and LQCD estimates. We have also obtained masses by using a lattice QCD inspired three-body confinement potential. The variational wave functions have been employed to compute the overlap integrals needed to evaluate the relevant Isgur-Wise functions that describe these decays. We have checked that our calculations are consistent with HQSS and have used them to estimate the semileptonic decay widths.

We have in addition made approximate, but model independent, predictions for ratios of semileptonic $b \rightarrow c$ decay widths based on the HQSS relations derived here, which we expect to be accurately fulfilled.

\section{ACKNOWLEDGMENTS}

This research was supported by DGI and FEDER funds, under Contracts No. FIS2011-28853-C02-02 and No. FPA2010-21750-C02-02, and the Spanish Consolider-Ingenio 2010 Programme CPAN (CSD200700042), by Generalitat Valenciana under Contract No. PROMETEO/20090090, and by the EU HadronPhysics2 project, grant agreement No. 227431.
[1] P. Hasenfratz, R. R. Horgan, J. Kuti, and J. M. Richard, Phys. Lett. 94B, 401 (1980).

[2] J. D. Bjorken, Report No. FERMILAB-Conf-85/69.

[3] B. Silvestre-Brac, Few-Body Syst. 20, 1 (1996).

[4] J. Vijande, H. Garcilazo, A. Valcarce, and F. Fernandez, Phys. Rev. D 70, 054022 (2004).

[5] W. Roberts and M. Pervin, Int. J. Mod. Phys. A 23, 2817 (2008).

[6] A. P. Martynenko, Phys. Lett. B 663, 317 (2008).

[7] X.H. Guo, K. W. Wei, and X.H. Wu, Phys. Rev. D 78, 056005 (2008).

[8] N. Brambilla, A. Vairo, and T. Rosch, Phys. Rev. D 72, 034021 (2005).

[9] N. Brambilla, J. Ghiglieri, and A. Vairo, Phys. Rev. D 81, 054031 (2010).

[10] J. R. Zhang and M. Q. Huang, Phys. Lett. B 674, 28 (2009).

[11] Y. Jia, J. High Energy Phys. 10 (2006) 073.

[12] F. J. Llanes-Estrada, O. I. Pavlova, and R. Williams, arXiv:1111.7087.

[13] S. Meinel, Phys. Rev. D 82, 114514 (2010).

[14] S. P. Baranov and V. L. Slad, Phys. At. Nucl. 67, 808 (2004).

[15] V. A. Saleev, Mod. Phys. Lett. A 14, 2615 (1999).
[16] M. A. Gomshi Nobary, Phys. Lett. B 559, 239 (2003); 598, 294(E) (2004).

[17] M. A. Gomshi Nobary and R. Sepahvand, Phys. Rev. D 71, 034024 (2005).

[18] M. A. Gomshi Nobary and R. Sepahvand, Nucl. Phys. B741, 34 (2006).

[19] Y.-Q. Chen and S.-Z. Wu, J. High Energy Phys. 08 (2011) 144; 09 (2011) 089(E).

[20] M. J. White and M. J. Savage, Phys. Lett. B 271, 410 (1991).

[21] E.E. Jenkins, M.E. Luke, A. V. Manohar, and M.J. Savage, Nucl. Phys. B390, 463 (1993).

[22] A. V. Manohar and M. B. Wise, Heavy Quark Physics, Cambridge Monographs on Particle Physics, Nuclear Physics, and Cosmology Vol. 10 (Cambridge University Press, Cambridge, England, 2000), ISBN 0-521-64241-8.

[23] M. A. Sanchis-Lozano, Nucl. Phys. B440, 251 (1995).

[24] C. Albertus, J. E. Amaro, E. Hernandez, and J. Nieves, Nucl. Phys. A740, 333 (2004).

[25] C. Albertus, E. Hernandez, J. Nieves, and J. M. VerdeVelasco, Eur. Phys. J. A 31, 691 (2007); 36, 119(E) (2008).

[26] C. Albertus, E. Hernandez, and J. Nieves, Phys. Rev. D 71, 014012 (2005).

[27] E. Hernandez, J. Nieves, and J. M. Verde-Velasco, Phys. Lett. B 663, 234 (2008). 
[28] C. Albertus, E. Hernandez, and J. Nieves, Phys. Lett. B 683, 21 (2010).

[29] C. Albertus, E. Hernandez, and J. Nieves, Phys. Lett. B 704, 499 (2011).

[30] A. F. Falk, H. Georgi, B. Grinstein, and M. B. Wise, Nucl. Phys. B343, 1 (1990).

[31] J. M. Flynn and J. Nieves, Phys. Rev. D 76, 017502 (2007); 77, 099901(E) (2008).

[32] A. F. Falk, Nucl. Phys. B378, 79 (1992).
[33] K. Nakamura et al. (Particle Data Group), J. Phys. G 37, 075021 (2010).

[34] C. Semay and B. Silvestre-Brac, Z. Phys. C 61, 271 (1994).

[35] T. T. Takahashi, H. Matsufuru, Y. Nemoto, and H. Suganuma, Phys. Rev. Lett. 86, 18 (2001).

[36] T. T. Takahashi, H. Suganuma, Y. Nemoto, and H. Matsufuru, Phys. Rev. D 65, 114509 (2002).

[37] G. S. Bali, C. Schlichter, and K. Schilling, Phys. Rev. D 51, 5165 (1995). 